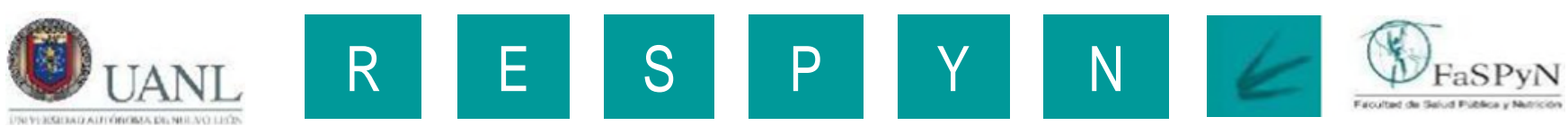 \\ Revista Salud Pública y Nutrición
}

\section{INTERVENCION NUTRICIONAL A PACIENTES CON ENFERMEDAD DE PARKINSON}

NUTRITIONAL INTERVENTION TO PATIENTS WITH PARKINSON DISEASE

Flores Solís María Dolores ${ }^{1}$, Gómez García Anel². Valenzuela Gandarilla Josefina ${ }^{3}$, Ibarra Bravo Octavio Miguel ${ }^{4}$, Punzo Bravo Guillermo 5 .

Facultad de Enfermería, U.M.S.N.H. Servicio de Nutricion Enteral y Parenteral del Hospital general Dr. Miguel Silva, SSM, ${ }^{2} \mathrm{IMSS}$, Morelia. Michoacán, ${ }^{3}$ Facultad de Enfermería, U.M.S.N.H., ${ }^{4}$ Hospital General

Dr. Miguel Silva, SSM, Medicina interna, Neurología, ${ }^{5}$ Hospital General Dr. Miguel Silva, SSM, Investigacion Clínica y Neurología

Citation: Flores Solís M.D., Gómez García A. Valenzuela Gandarilla J., Ibarra Bravo O. M., Punzo Bravo G. (2017) Intervención Nutricional en Pacientes con Enfermedad de Parkinson, Revista de Salud Pública y Nutrición, 16(2), 28-35.

Editor: Esteban G. Ramos Peña, Dr. CS., Universidad Autónoma de Nuevo león, Facultad de Salud Pública, Monterrey Nuevo León, México.

Copyright: 02017 Flores Solís M.D. et al. This is an open-access article distributed under the terms of Creative Commons Attribution License [CC BYND 4.0], which permits unrestricted use, distribution, and reproduction in any medium, provided the original author and source are credited.

Competing interests: The authors have declared that no competing interests exist.

DOI: https://doi.org/10.29105/respyn16.2-4

*Email: doloresfloresnut@hotmail.com. 


\title{
INTERVENCION NUTRICIONAL A PACIENTES CON ENFERMEDAD DE PARKINSON
}

\author{
Flores Solís María Dolores', Gómez García Anel². Valenzuela Gandarilla Josefina³, Ibarra Bravo Octavio \\ Miguel $^{4}$, Punzo Bravo Guillermo 5 .
}

\author{
Facultad de Enfermería de la Universidad Michoacana de San Nicolás de Hidalgo. ${ }^{2}$ Investigadora IMSS, Morelia. \\ Michoacán. ${ }^{3}$ Facultad de Enfermería, U.M.S.N.H. ${ }^{4}$ Hospital General Dr. Miguel Silva, SSM, Medicina interna, Neurología. \\ ${ }_{5}$ Hospital General Dr. Miguel Silva, SSM, Investigacion Clínica y Neurología.
}

\section{RESUMEN}

Introducción: Los pacientes con Enfermedad de Parkinson (EP) llegan a tener carencia nutrimental debida a incremento en el gasto energético, temblor y rigidez, disminución de ingesta alimentaria, falta de apetito, náuseas/vómito, problemas de deglución, sensación de saciedad temprana, aporte calórico inadecuado de nutrientes en la dieta, constipación y estreñimiento. Objetivo: Realizar una intervención nutricional para monitorear el estado nutricional con el uso de complementos nutricionales, dieta polimérica, fibra soluble e insoluble, antioxidantes y espesantes a pacientes con enfermedad de Parkinson. Métodos: Estudio longitudinal, se realizó de Junio 2016 a Junio 2017 a una muestra de 54 pacientes con EP que estuvieron todo el estudio y acudían a la consulta de neurología del Hospital General Dr. Miguel Silva, SSM, se les proporcionó dieta polimérica, fibra soluble e insoluble, antioxidantes y espesantes así como orientación alimentaria en su dieta habitual. El monitoreo se llevó a cabo con Evaluación Nutricional: Antropométricos, Bioquímicos, Clínicos y Dietéticos. Resultados: Se valoró nutricionalmente a 38 pacientes hombres y 16 mujeres de entre 30-85 años con EP., la disfagia fue la que mejor respuesta tuvo al tratamiento nutricio con 45 puntos porcentuales menos al final respecto al inicial, le sigue el estreñimiento con 31.5 puntos porcentuales, el $74.3 \%$ de los pacientes mantuvieron sus valores de CMB al final respecto al inicial, el resto de ellos aumentaron sus valores. Conclusiones: Se observó incremento en el peso en masa muscular así como mejor funcionamiento gastrointestinal, mejor estado nutricional, tendencia a mejor calidad de vida.

Palabras Clave: Intervención nutricional, Parkinson.

\section{ABSTRACT}

Introduction: Patients with Parkinson's disease (PD) have nutritional deficiency due to increased energy expenditure, tremor and stiffness, decreased food intake, poor appetite, nausea / vomiting, swallowing problems, early satiety, caloric intake inadequate nutrients in the diet, constipation and constipation. Objectives: To perform a nutritional intervention to monitor nutritional status with the use of nutritional supplements, polymeric diet, soluble and insoluble fiber, antioxidants and thickeners to patients with Parkinson's disease. Methods: A longitudinal study was conducted from June 2016 to June 2017 in a sample of 54 patients with PD who were in the study and attended the neurology clinic of the General Hospital Dr. Miguel Silva, SSM. They were given a polymeric diet, fiber Soluble and insoluble, antioxidants and thickeners as well as dietary guidance in their usual diet. The monitoring was carried out with Nutritional Assessment: Anthropometric, Biochemical, Clinical and Dietetic. Results: A total of 38 male and 16 female patients aged 30-85 years with PD were nutritionally evaluated. Dysphagia was the one with the highest nutritional response, 45 percentage points lower than the initial one, followed by constipation with 31.5 Percentage points, $74.3 \%$ of the patients maintained their CMB values at the end of the initial one, and the rest of them increased their values. Conclusions: It was observed an increase in muscle mass as well as better gastrointestinal function, better nutritional status, and tendency to better quality of life. Values.

Key words: Nutritional intervention, Parkinson. 


\section{Introducción}

La enfermedad de Parkinson (EP) es un trastorno degenerativo del sistema nervioso central. Fue descrita por primera vez en 1817 por James Parkinson, un médico británico que publicó un artículo sobre lo que llamó "la parálisis temblorosa." La EP pertenece a un grupo de enfermedades llamadas trastornos del movimiento. Se caracteriza por una pérdida de las células de la sustancia nigra y una deficiencia de dopamina en el cuerpo estriado. La dopamina no atraviesa la barrera hematoencefálica, por lo que no puede administrarse directamente para suplir esta deficiencia.

Una forma de hacerlo es como levodopa, profármaco que sí atraviesa la barrera hematoencefálica y que, una vez en el cerebro, se convierte en dopamina. El uso de levodopa en la EP comienza en el año 1961 y, aunque han pasado más de 50 años continúa siendo el principal fármaco para su tratamiento, junto con compuestos carbidopa o bencerazida (inhibidores de la dopadescarboxilasa), que evitan su rápida degradación y se consigue que la cantidad de levodopa que alcanza el sistema nervioso central sea mayor aumentando el tiempo de semivida de la levodopa. Aunque la levodopa es el fármaco más eficaz para el tratamiento sintomático del Parkinson, a medida que progresa la enfermedad y aumenta el tiempo de tratamiento, también se incrementa el porcentaje de pacientes que presentan complicaciones. Los cuatro síntomas principales son temblor, o temblor en las manos, los brazos, las piernas y la mandíbula o la cabeza; rigidez, o agarrotamiento de las extremidades y el tronco; bradicinesia, o lentitud en los movimientos; e inestabilidad postural, o deterioro del equilibrio. Estos síntomas generalmente comienzan gradualmente y empeoran con el tiempo. (Plana M. 2009).

A medida que los síntomas se vuelven más pronunciados, los pacientes pueden tener dificultad para caminar, hablar o completar otras tareas sencillas como comer.

La EP es crónica, degenerativa y progresiva, que persiste durante un extenso período de tiempo, lo que significa que sus síntomas empeoran con el tiempo. No es contagiosa. Aunque algunos casos de Parkinson parecen ser hereditarios y otros pueden rastrearse a mutaciones genéticas específicas.
Muchos investigadores ahora creen que la enfermedad es consecuencia de una combinación de susceptibilidad genética y exposición a uno o más factores ambientales que desencadenan la enfermedad, otros estudios realizados demuestran que el $24 \%$ de los pacientes con EP padecen desnutrición y el $60 \%$ tiene riesgo de padecerla.

La intervención nutricional adecuada al inicio de la EP y el monitoreo continuo para el apego al tratamiento médico y nutricional evitan un deterioro de la masa y la fuerza muscular para prevenir la Sarcopenia en este tipo de pacientes. (Burgos R. Virgili 2009)

El deterioro del estado nutricional es multifactorial en la EP como es debido a un incremento en el gasto energético por la enfermedad, disminución de la ingesta alimentaria por falta de apetito, náuseas o ayunos, problemas de deglución, sensación de saciedad temprana, un aporte calórico inadecuado de nutrientes en la dieta, estos pacientes a menudo experimentan cambios en el peso corporal durante el curso de la enfermedad, tanto la pérdida de peso y aumento de peso pueden ocurrir, pero la pérdida de peso es más frecuente y se asocia con aumento de la discinesia, mayor mortalidad donde se pierde más masa magra muscular como proteína visceral por lo que es importante llevar estos registros y evitar la progresión acelerada de la enfermedad y pobre calidad de vida; (Sharma y Vassallo 2014) encontraron pruebas que vinculan la pérdida de peso con una progresión más rápida del Parkinson en su fase inicial, mientras que un leve aumento de masa corporal en los pacientes parece frenar el avance de la enfermedad.

Es importante reconocer la interacción fármaconutriente ya que la Levodopa tiene interacción con la proteína y compiten en las vías metabólicas por su absorción por lo que no se debe de administrar con alimentos de origen animal. La levodopa oral se absorbe rápidamente en el intestino delgado a través del sistema de transporte para aminoácidos aromáticos. Los aminoácidos de la dieta pueden alterar su absorción. Las concentraciones máximas del fármaco en plasma suelen ocurrir entre $1 / 2$ y 2 horas después de una dosis oral. La vida media en plasma es de 1 a 3 horas.

El objetivo fue realizar una intervención nutricional para monitorear el estado nutricional con el uso de 
complementos nutricionales, dieta polimérica, fibra soluble e insoluble, antioxidantes y espesantes a pacientes con enfermedad de Parkinson.

\section{Material y Métodos}

Se hizo un estudio longitudinal, cuasi experimental, se realizó de Junio 2016 a Junio 2017 a una muestra de 54 pacientes con EP que estuvieron todo el estudio y acudían a la consulta de neurología del Hospital General Dr. Miguel Silva, SSM, estuvo formada por 38 hombres y 16 mujeres, con un rango de edad entre 30-85 años que se encuentran en el estadio I-V, los cuales se les evalúo su estado nutricional utilizando los indicadore: antropométricos, bioquímicos, clínicos y dietéticos, comparando con tablas de referencia y valores de laboratorio para su adecuada interpretación. Los estándares de referencia de la circunferencia muscular libre de grasa $\mathrm{CMB}=\mathrm{PB}(\mathrm{cms})-.(0.31 \mathrm{X}$ PT $(\mathrm{cm})$ ), el cual es un indicador nutricional objetivo y con una especificidad alta, es medible, fácil de realizar y tener un monitoreo continuo.

El CONUT (Control Nutricional) es una herramienta que nos indica, la alerta baja, modera o severa del estado nutricional con mediciones específicas, valorando el grado de desnutrición monitoreando proteína visceral y reserva de colesterol. (Ulíbarri y Col. 2005), se aprecia en (tablas 1 y 2).

Tabla 1. Estándares de referencia para la circunferencia Muscular del Brazo

\begin{tabular}{ll}
\hline Grado de Medición & $\begin{array}{c}\text { GENERO } \\
\text { Hombres Mujeres }\end{array}$ \\
\hline Estándar & $25.3 \mathrm{~cm} 23.2 \mathrm{~cm}$ \\
$90 \%$ del estándar & $22.8 \mathrm{~cm} 20.9 \mathrm{~cm}$ \\
$80 \%$ del estándar & $20.2 \mathrm{~cm} 18.6 \mathrm{~cm}$ \\
$70 \%$ del estándar & $17.7 \mathrm{~cm} 16.2 \mathrm{~cm}$ \\
$60 \%$ del estándar & $15.2 \mathrm{~cm} 13.9 \mathrm{~cm}$ \\
\hline
\end{tabular}

TABLAS DE FRISANCHO 1990 Y CONSENSO FELANPE 2008

Tabla 2.- Valoración del grado de desnutrición

\begin{tabular}{lrrrr}
\hline Parámetro & Normal & Leve & Moderado & Severa \\
\hline Albumina (gr/dl) & $3.5-4.5$ & $3-3.49$ & $2.5-2.9$ & $<2.5$ \\
Puntuación & 0 & 2 & 4 & 6 \\
Cuenta total de Linfocitos $(\mathrm{ml})$ & $>1,600$ & $1200-1599$ & $800-1200$ & $<800$ \\
Puntuación & 0 & 1 & 2 & 3 \\
Colesterol (mg/dl) & $>180$ & $140-180$ & $100-139$ & $<100$ \\
Puntuación & 0 & 1 & 2 & 3 \\
Total del filtro & $0-1$ & 42827 & 42952 & $>8$ \\
\hline Fuente: J. Ignacio de Ulíbarr y Col. CONUT. Nutr. Hosp. (2005) XX (1) 38-45 & & &
\end{tabular}

El criterio de inclusión fue: Pacientes con EP que acudieran a la consulta de neurología, HGMS de Junio del 2016 a Junio del 2017. Los criterios de exclusión fueron: pacientes qué se encontraban hospitalizados, pacientes que no acepten realizarse las pruebas o participar en la encuesta alimentaria, se contó con la misma muestra de pacientes con EP al inicio y al final de la Intervención Nutricional. Se tomaron en cuenta como criterios de eliminación: pacientes que no cumplieran en realizarse los exámenes de laboratorio o no tuvieran el apego a la orientación nutricional $\mathrm{y}$ al tratamiento con suplementos fibra y espesantes.

Clínicamente se revisó a los pacientes con EP y se estuvieron analizando los síntomas como nauseas, vomito, anorexia, disfagia, problemas dentales, estreñimiento, temblor/marcha, observando con que frecuencia lo padecían antes y durante la Intervención nutricional y/o con el apoyo de complementos nutricionales en su dieta diaria, orientando al paciente y su familia en la adecuada ingesta alimentaria.

Se obtuvo el consentimiento informado para la intervención nutricional que fue mejorando su dieta habitual o incluyendo dieta polimérica, fibra soluble o insoluble, antioxidantes y espesantes en el caso de una marcada deglución.

Para el análisis de la información se utilizó el programa estadístico SPSS V.20 y Excel.

\section{Resultados}

La población estudiada corresponde a pacientes que tienen diagnóstico de Enfermedad de Parkinson, el $70.4 \%$ fueron (38) hombres y el $29.6 \%$ (16) mujeres. $\mathrm{El}$ mayor porcentaje 52\%, (el cual el 33\% hombres y $19 \%$ mujeres) correspondió a pacientes de 40 a 59 años de edad (ver tabla 3).

Tabla 3. Pacientes con EP por género según edad

\begin{tabular}{lccc}
\hline & \multicolumn{2}{c}{ Género } & \\
\cline { 2 - 3 } & Hombres $(n=38)$ & Mujeres $(n=16)$ & Total \\
\hline Edad & $\%$ & $\%$ & $\%$ \\
$20-39$ & 9.0 & 0.0 & 4.0 \\
$40-59$ & 33.0 & 19.0 & 52.0 \\
$60-79$ & 21.0 & 8.0 & 29.0 \\
$80-99$ & 7.0 & 3.0 & 10.0 \\
\hline
\end{tabular}

Fuente: Directa 
En cuanto al estadio de la enfermedad, el $16 \%$ tenía estadío I, el $34.3 \%$ estadío II, $19.7 \%$ estadío III, $24.4 \%$ estadío IV y el $5.6 \%$ estadío V.

Referente al riesgo nutricio (Índice de Naber) que presentaron los pacientes, el $30.8 \%$ sin riesgo, $15.4 \%$ desnutrición leve, $38.5 \%$ desnutrición moderada, $15.3 \%$ desnutrición severa.

De acuerdo al grado de desnutrición por albúmina, el $56.9 \%$ de los pacientes con EP estudiados tuvieron grado normal, $23.7 \%$ desnutrición leve, $11.7 \%$ desnutrición moderada y el $7.7 \%$ desnutrición severa.

La medición de la circunferencia muscular que normalmente realiza el Nutriólogo tiene validez cuando se lleva el monitoreo periódico y con apego al tratamiento nutricional. Se realizó la medición de la $\mathrm{CMB}$ en el inicio y en el seguimiento. Como se observa en la tabla 4 , el $74.3 \%$ de los pacientes mantuvieron sus valores al final respecto al inicial, el resto de ellos aumentaron sus valores. El mayor porcentaje (51.9\%, IC: 38.6-65.2) se mantuvieron en normal, ningún paciente disminuyó sus valores iniciales al final del estudio.

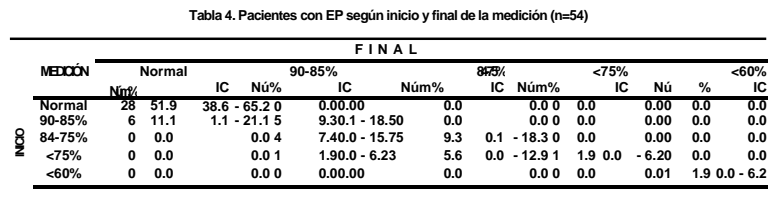

En esta tabla 5, se observa una tendencia a la mejoría del estado nutricio al final del estudio, en el nivel normal mostró una tendencia al aumento del porcentaje, mientas en los demás estados de nutrición la tendencia fue a la disminución al final del estudio, lo anterior nos habla de que el estado nutricio en estos pacientes muestra una tendencia positiva con tratamiento nutricional.

Tabla 5. Pacientes* por Valoración inicial y final según del grado de desnutricion , CONUT

\begin{tabular}{lrrrr}
\hline & \multicolumn{2}{c}{ Inicial } & \multicolumn{2}{c}{ Final } \\
\hline & $\%$ & \multicolumn{1}{c}{ IC } & $\%$ & IC \\
\hline Normal & 33.4 & $20.8-46.0$ & 51.4 & $38.1-64.7$ \\
Desnutrición leve & 27.6 & $15.7-39.5$ & 23.3 & $12.0-34.6$ \\
Desnutrición moderada & 22.6 & $11.4-33.8$ & 14.2 & $4.9-23.5$ \\
Desnutrición severa & 16.4 & $6.5-26.3$ & 11.1 & $2.7-19.5$ \\
\hline
\end{tabular}

Fuente: Directa
$n=54$
En la tabla 6, pacientes con EP por evaluación inicial y final según presencia de sintomatología, la disfagia fue la que mejor respuesta tuvo al tratamiento nutricio con 45 puntos porcentuales menos al final respecto al inicial, le sigue el estreñimiento con 31.5 puntos porcentuales. Todas las demás sintomatologías tienen tendencia de disminuir el porcentaje de pacientes con sintomatología al final de tratamiento respecto al inicio.

Tabla 6. Pacientes* con EP por evaluación inicial y final según presencia de sintomatología

\begin{tabular}{lccrr}
\hline & \multicolumn{1}{l}{ INICIO } & \multicolumn{1}{c}{ FINAL } \\
\hline INICIO & \multicolumn{1}{l}{$\%$ IC } & \multicolumn{1}{c}{$\%$ IC } \\
\hline Nauseas/vòmito & 35.2 & $22.5-47.9$ & 11.1 & $2.7-19.5$ \\
Disfagia & 66.7 & $54.1-79.3$ & 21.7 & $10.7-32.7$ \\
Anorexia & 46.3 & $33.0-49.6$ & 24.1 & $12.7-35.5$ \\
Dentadura & 77.8 & $66.7-88.9$ & 57.4 & $44.2-70.6$ \\
Estreñimiento & 74.1 & $62.4-85.7$ & 42.6 & $29.4-55.8$ \\
Temblor-marcha & 72.2 & $60.3-84.1$ & 59.3 & $46.2-72.4$ \\
\hline Fuente: Directa & & &
\end{tabular}

${ }^{*} \mathrm{n}=\mathbf{5 4}$

\section{Discusión}

La enfermedad de Parkinson (EP) ocupa el segundo lugar dentro de las demencias más comunes, luego de la enfermedad de Alzheimer (EA). Afecta por lo general a personas mayores de 50 años, con un incremento de la incidencia después de los 60 años de edad. La prevalencia de EP, reportada a nivel mundial en las personas mayores de 65 años, es de aproximadamente del $1 \%$, con una incidencia mundial cruda ajustada por edad de 9,7 a 13,8 casos por cada 100.000 personas por año. (Sheard, 2011). Las enfermedades de Parkinson y Alzheimer son consideradas factores de riesgo de malnutrición energético proteica. Es esencial la detección precoz de estas situaciones de riesgo para contribuir al desarrollo de estrategias de intervención nutricional, retardando de este modo la aparición de comorbilidades asociadas que comprometan el estado de salud del paciente y su calidad de vida. (Camina, 2013).

La desnutrición calórica proteica y proteica son las causas más frecuentes de las alteraciones nutricionales en el anciano y uno de los grandes síndromes geriátricos que llevan a la incapacidad. Esta alta frecuencia está motivada por los numerosos factores de riesgo de esta población. Se produce una 
disminución de la masa corporal magra (6,32 en cada década a partir de los 30 años). Este decremento se llama Sarcopenia y tiene como consecuencia la pérdida de fuerza, capacidad aeróbica y funcionalidad. La Sarcopenia es multifactorial y está relacionada con el estrés oxidativo, habiéndose considerado como más importantes la alteración en la síntesis y degradación de las proteínas, los procesos inflamatorios, las alteraciones hormonales y la disfunción mitocondrial (Cruz-Jentoft \& Cuesta, 2011). Durante el proceso de envejecimiento se tiene relación con obesidad, osteoporosis y enfermedades metabólicas (Rieu, 2006).

Por estas consecuencias fue importante evaluar el efecto de la ingesta proteica con la masa muscular del brazo y las proteínas viscerales con el instrumento CONUT, así como mejorar la sintomatología. (Camina, 2013).

Actualmente no existe un patrón de oro en la valoración del paciente con Parkinson y el CONUT (Control Nutricional) es una herramienta que nos ayuda a valorar el grado de desnutrición con una especificidad y sensibilidad alta, con la obtención de Albumina sérica, cuenta total de linfocitos y colesterol son datos que alertan en la desnutrición leve, moderada o severa para implementar una intervención nutricional temprana y adecuada, evitando complicaciones de malnutrición o desnutrición que son más difíciles de rehabilitar en pacientes con EP, es importante monitorear las proteínas viscerales para mejorar su aporte nutricional. (Conut, 2005)

El aumento de peso a veces se observa en las etapas iniciales de la enfermedad y probablemente dependiente del tratamiento dopaminérgico, lo que mejora los síntomas del motor y puede modular la conducta alimentaria (Sharma, 2014). Pero la pérdida de peso es más frecuente y se asocia con aumento de la discinesia, mayor mortalidad, progresión acelerada de la enfermedad y pobre calidad de vida. Las causas de pérdida de peso en la EP aún no están claras. Especialmente cambios en el gasto de energía y conducta alimentaria es por eso que en este estudio se monitoreo cada 2 meses a los pacientes para tener un apego en la orientación alimentaria y en la utilización adecuada de los complementos como fue la dieta polimérica, antioxidantes, fibra soluble e insoluble y espesantes.(Kistner, 2014)
"Los pacientes EP que experimentaron temprana pérdida de peso resultaron tener formas más severas y sistemáticas de la enfermedad, posiblemente debido a la implicación del sistema neuroendocrino o el sistema gastrointestinal nervioso, mientras aquellos que ganaron peso tuvieron una versión más leve de la enfermedad", explicó Anne-Marie Wills, Catedrática. Neurólogos del Massachusetts General Hospital (MGH).

La restricción proteica constituye una práctica conocida para tratar algunas enfermedades, pero supone el riesgo de exponer al paciente a un aporte insuficiente de proteínas. La levodopa compite con aminoácidos de cadena larga por transportadores a nivel gastrointestinal, barrera hematoencefálica y empeoramiento de la sintomatología motora. Es recomendable asegurar un aporte mínimo en proteínas de $0,8 \mathrm{~g} / \mathrm{kg} / \mathrm{día}$ en pacientes con circunferencia muscular del brazo normal y proteínas viscerales normales, pero aquellos pacientes que presenten déficit de circunferencia muscular del brazo, albumina y cuenta total de linfocitos bajas se requiere aumentar la proteína hasta $1.5 \mathrm{~g} / \mathrm{kg} /$ día y ser posible, de alto valor biológico, el cuidado que se debe de tener es que deben pasar de 2 a 3 horas de que ingiera la Levodopa. Puede realizarse una redistribución horaria de la ingesta de proteínas, restringiéndola durante el día y cubriendo las raciones recomendadas por la noche (cena). (Lara, 2013).

Debe observarse la dentadura de los enfermos ya que se deteriora más que cualquier persona porque aprietan mucho los dientes y se caen con más rápidez las piezas dentales, se debe realizar la interconsulta con el odontólogo para mejorar su dentadura y su ingesta alimentaria.

Se recomienda la administración de suplementos dieta polimérica, antioxidantes, fibra soluble e insoluble y espesantes a media mañana, con la merienda y antes de acostarse, ya que es cuando menos alteran el apetito de las principales comidas. Asimismo, debe distribuirse la ingestión de proteínas a lo largo del día para optimizar su ingesta, al contrario que en las personas jóvenes, en los que se optimizan en la comida principal. (Shen, 2015)Se indica que la retirada de estos suplementos debe realizarse cuando haya desaparecido la situación 
causante como los pacientes que mejoraron la deglución fueron poco a poco dejando los espesantes, los pacientes que presentaron estreñimiento severo se fue incrementando la fibra soluble e insoluble hasta mejora el tránsito intestinal, sin embargo no se ha dejado la fibra en su alimentación diaria, se orientó al paciente y su familia para adecuar su ingesta por medio de papillas e introduciendo la fibra a la comida diaria en sopas, ensaladas, guisos, gelatinas, compota de frutas, licuados, etc., el consejo nutricional es muy valioso en las diferentes etapas de la enfermedad, el Nutriólogo es el profesional indicado para mejorar la alimentación diaria de los enfermos con Parkinson y mantener o mejor su estado nutricional.

La atrofia de las papilas gustativas conduce a la selección de alimentos con sabores dulces y salados (por ejemplo, productos azucarados o fuertemente sazonados. Asimismo, la pérdida de sensibilidad gustativa puede conllevar menor ingesta de nutrientes y reducción del apetito, por lo que fue importante mejorar su ingesta con alimentos suaves y espesantes. Tragar es una serie de eventos coordinados secuenciales que asegura el paso de cualquier sustancia (alimentos, líquidos, saliva, moco, drogas) desde la boca hasta el estómago a través de la faringe y el esófago, evitando el paso de la sustancia tragada hacia las vías respiratorias, si esto se complica se denomina disfagia es el término usado para describir cualquier dificultad en la deglución; por lo tanto, la disfagia no representa un diagnóstico médico, sino un síntoma que es muy característico en la EP y debe ser detectada tempranamente aumentando su actividad con espesantes para evitar se atrofie la deglución y el EP requiera sonda de Gastrostomía para su alimentación.(Hughes, 2002)

También estos enfermos cursan con trastornos motores gastrointestinales provocando estreñimiento que llega a ser severo de 8 a 13 días para la defecación y mucho es también por la falta de ingesta adecuada de líquidos por problemas de deglución, esto se trató con fibra soluble e insoluble que se fue incrementando poco a poco en la dieta hasta mejorar el tránsito intestinal. Beneficios de la ingesta de fibra soluble se disuelve en el líquido se formara un gel es más viscoso y en el color se forman ácidos grasos de cadena corta (AGCC) estimulando y fortaleciendo la flora intestinal mejorando la absorción de calcio, magnesio y fosforo, regulan los niveles de glucosa en sangre, bajan el colesterol LDL y menor riesgo de enfermedad cardiaca, en especial la Inulina quien tiene más efectividad prebiótica así como los fructo oligosacáridos. La fibra insoluble requiere mayor cantidad de líquidos ya que se expande y fortalece las vellosidades (ejercita en su paso) y aumenta el tránsito intestinal eliminando las sustancias de desecho, estos beneficios son muy recomendables para los pacientes con Parkinson.

El temblor y la marcha en la EP representan un gasto del $30 \%$ al $50 \%$ del Gasto Energético Basal por lo que el apego al tratamiento médico y nutricional es fundamental.

Puede establecerse que las tasas de mortalidad por EP son bajas para cualquier estrato de edad y que la edad de la muerte de los pacientes ha ido desplazándose hasta edades más avanzadas, por un progresivo aumento de la esperanza de vida. Además, todo parece indicar que el tratamiento con levodopa reduce el riesgo de muerte de los pacientes, especialmente durante los primeros años de la enfermedad, por lo que es importante la intervención nutricional y que el Nutriólogo Clínico tiene un campo importante y líneas de investigación en la Neurología.

\section{Conclusiones:}

La EP es degenerativa y progresiva con muchos matices y sintomatologías que es un reto importante para la Nutriología. No existe un "patrón de oro" para la evaluación nutricional y es importante valorar la masa muscular y la masa visceral porque es lo que más pierde el enfermo de Parkinson. La intervención nutricional debe ser temprana al diagnóstico para evitar deterioro en los pacientes, proporcionando orientación alimentaria continua para que se llegue al apego en la ingesta adecuada de alimentos, nutrientes, y complementos nutricionales (dieta polimérica con fibra soluble e insoluble, antioxidantes y espesantes) en su dieta habitual, esto refleja en el paciente con Parkinson un mejor estado nutricional, mejor respuesta al tratamiento farmacológico y mejor calidad de vida.

Recomendar horarios de administrar la Levodopa y horarios de Alimentación a la ingesta de proteínas para que ambos tengan sus rutas de aprovechamiento y se vea reflejado en la evolución de la salud. 
La malnutrición y desnutrición leve, moderada o severa ya que es más difícil rehabilitar el estado nutricional.

La Disfagia está muy presente en pacientes con Parkinson, se requiere evaluar desde el diagnóstico para activar la deglución evitando o retrasando el uso de sondas como Gastrostomía para su alimentación. La combinación de la fibra soluble e insoluble tiene más beneficios en el enfermo de Parkinson que solo darle fibra insoluble o laxantes.

\section{Bibliografía}

A. Leopold DO, Marion C. Kagel MA (1996) Prepharyngeal dysphagia in Parkinson's disease. Dysphagia 11(1):14-22

Argolo N, Sampaio M, Pinho P, Melo A, Nobrega AC (2015) Videofluoroscopic predictors of penetrationaspiration in Parkinson's Disease patients. Dysphagia 30:751-758

Burgos R. Virgili N. (2009). Role Of Nutrition In Prevention And Course Of Neurodegenerative Diseases. Suplemento de Nutrición Hospitalaria. Volumen (2) 2 Mayo 2009 SENPE- Nutrición Española de nutrición parenteral y Enteral.

Camina Martín, MA., de Mateo Silleras, B., Carreño Enciso, L., et al. Cambios en la composición corporal en función del grado de demencia en un grupo de ancianos institucionalizados. Nutr Hosp. 2013; 28(3): 1093-1101

Castillo, JC. Gómez, A. Velasco, N. et al. (2016) Nutritional assessment of hospitalized patients in Latin America: association with prognostic variables. The ENHOLA study. Nutr Hosp. 33(3):655-662 ISSN 02121611 - CODEN NUHOEQ S.V.R. 318

Castro, G. Méndez C. Suverza A. (2009). Nutrición en la Práctica Clínica. México: Alfil.

CONUT: J. Ignacio de Ulíbarri*, A. González-Madroño. A tool for Controlling Nutritional Status. First validation in a hospital population. Nutr. Hosp. (2005) XX (1) 3845 ISSN 0212-1611.

Cruz-Jentoft AJ. Cuesta F, G.-C. M.-S. (2011). La ecolosión de la sarcopenia . Original, Sociedad Española de geriatriá y gerontología , Observatorio de Sarcopenia, España.

Escott-Stump S. (2005). Nutrición, diagnóstico y tratamiento. ( $5^{\mathrm{a}}$ Ed.) México: Ed. McGraw-Hill.
Ethem Murat Arsava Editor Nutrition in Neurologic Disorders A Practical Guide, ISBN 978-3-319531700 ISBN 978-3-319-53171-7 (eBook) DOI 10.1007/978-3-319-53171-7 Library of Congress Control Number: 2017939690 (c) Springer International Publishing AG 2017.

Fereshtehnejad SM, Ghazi L, Shafieesabet M, Shahidi GA, Delbari A, Lo J (2014) Motor, psychiatric and fatigue features associated with nutritional status and its effects on quality of life in Parkinson's Disease Patients. PLOS One 9(3):1-10

Goates S, Du K, Braunschweig CA, Aransberg MB (2016) Economic burden of diseaseassociated malnutrition at the state level. PLoS One 21:1-15.

Hughes AJ, Daniel SE, Ben-Shlomo Y, Lees AJ. The accuracy of diagnosis of parkinsonian syndromes in a specialist movement disorder service. Brain. 2002; $125: 861-70$

Instituto Mexicano del Seguro Social. (2013). Guía de Práctica Clínica. Desnutrición Intrahospitalaria: Tamizaje, diagnóstico y tratamiento. México: Instituto Mexicano del Seguro Social.

Kaiser MJ, Bauer JM, Ramsch C, Uter W, Guigoz Y, Cederholm $\mathrm{T}$ et al (2010) Frequency of malnutrition in older adults: a multinational perspective using the mini nutritional assessment. J Am Geriatr Soc 58:1734- 1738

Kirschmann, J. Nutrition Search. (2008). Almanaque de Nutrición. $6^{\text {a }}$ Ed. México: McGraw-Hill.

Kistner A, Lhommee E, Krack P (2014) Mechanisms of body weight fluctuations in Parkinson's disease. Front Neurol 5:84

Lara Gonzalez S. (2013).Evaluación de buenas prácticas de manufactura en la elaboración de fórmulas enterales en hospitales públicos de Santiago, Chile Nutrición Hospitalaria. 2013; 28(6):2021-2026. SENPE-Nutrición Española de nutrición parenteral y enteral.

Nishioaka S, Okamoto T, Takayama T, Urushihara M, Watanabe M, Kiriya Y et al (2016) Malnutrition risk predicts recovery of full oral intake among older adult stroke patients undergoing enteral nutrition: secondary analysis of a multicentre survey (the APPLE study).
Clin
Nutr (ahead publ) doi:10.1016/j.clnu.2016.06.028

Nishioka S, Wakabayashi H, Nishioka E, Yoshida T, Mori $\mathrm{N}$, Watanabe R (2016) Nutritional improvement correlates with recovery of activities of daily living among malnourished elderly stroke patients in the 
convalescent stage: a cross-sectional study. JAcad Nutr Diet 116:837-843

Norma Oficial Mexicana NOM-043-SSA2-2012, (2012). Servicios Básicos de Salud. Promoción y Educación para la Salud en Materia Alimentaria. Criterios para Brindar Orientación. México: Secretaria de Salud.

Plana M. (2009). Relación entre el sistema nervioso y la nutrición Clínica. Suplemento, Nutrición Hospitalaria, Vol (2).

Saleem TZ, Higginson IJ, Chaudhuri KR, Martin A, Burman R, Leigh PN (2012) Symptom prevalence, severity and palliative care needs assessment using the Palliative Outcome Scale: A cross-sectional study of patients with Parkinson's disease and related neurological conditions. Palliative Medicine 27(8):722-731

Sharma JC, Vassallo M (2014) Prognostic significance of weight changes in Parkinson's disease: the Parkweight phenotype. Neurodegener Dis Manag 4:309316

Sheard JM, Ash S, Mellick GD, Silburn PA, Kerr Gk (2013) Markers of disease severity are associated with malnutrition in Parkinson's Disease. PLOS One $8(3): 1-8$

Sheard, JM., Ash, S., Silburn, PA., et al. Prevalence of malnutrition in Parkinson's disease: a systematic review. Nutrition Reviews 2011; 69(9): 520-532.

Shen L (2015) Associations between B vitamins and Parkinson's disease. Nutrients 7:7197-7208

Stephen J. McPhee, Maxine A. Papadakis. (2009) Diagnóstico clínico y tratamiento. (48ª Ed.) México: McGraw-Hill.

Thomas S, MacMahon D (2004) Parkinson's Disease, palliative care and older people, part II. Nursing Older People 16(2):22-26.

Walker RW, Churm D, Dewhurst F, Samuel M, Ramsell A, Lawrie C, et al (2014) Palliative care in people with idiopathic Parkinson's disease who die in hospital. BMJ Supportive \& Palliative Care 4:64-67 